\title{
Atlantic Diabetes in Pregnancy (DIP): the prevalence and outcomes of gestational diabetes mellitus using new diagnostic criteria
}

\author{
E. P. O'Sullivan • G. Avalos • M. O'Reilly • \\ M. C. Dennedy • G. Gaffney • F. Dunne • \\ on behalf of the Atlantic DIP collaborators
}

Received: 2 December 2010 / Accepted: 17 March 2011/Published online: 15 April 2011

(C) Springer-Verlag 2011

\begin{abstract}
Aims/hypothesis New diagnostic criteria for gestational diabetes mellitus (GDM) have recently been published. We wished to evaluate what impact these new criteria would have on GDM prevalence and outcomes in a predominantly European population.

Methods The Atlantic Diabetes In Pregnancy (DIP) programme performed screening for GDM in 5,500 women with an oral glucose tolerance test at 24-28 weeks. GDM was defined according to the new International Association of Diabetes and Pregnancy Study Groups (IADPSG) criteria and compared with previous WHO criteria; maternal and neonatal adverse outcomes were prospectively recorded.

Results Of the participants, $12.4 \%$ and $9.4 \%$ were diagnosed with GDM using IADPSG and WHO criteria, respectively. IADPSG GDM pregnancies were associated with a statisti-
\end{abstract}

Atlantic DIP collaborators (Ireland): L. Carmody, Galway University Hospital, Galway; M. Durkan, M. Brassil, Y. Mitchell, Portiuncla

Hospital, Ballinasloe, Co. Galway; M.S. Mohamed, M. Todd, Mayo

General Hospital, Castlebar, Co. Mayo; C. McHugh, H. Langan,

M. Keenan, Sligo General Hospital, Co. Sligo; M. Nandini,

T. Gallagher, Letterkenny General Hospital, Co. Donegal.

E. P. O’Sullivan · G. Avalos • M. O'Reilly • M. C. Dennedy •

F. Dunne

Department of Diabetes, Galway University Hospital,

Galway, Ireland

G. Gaffney

Department of Obstetrics and Gynaecology,

National University of Ireland,

Galway, Ireland

\section{F. Dunne $(\bowtie)$}

School of Medicine, Clinical Science Institute,

National University of Ireland,

Galway, Ireland

e-mail: fidelma.dunne@nuigalway.ie cally significant increased incidence of adverse maternal outcomes (gestational hypertension, polyhydramnios and Caesarean section) and neonatal outcomes (prematurity, large for gestational age, neonatal unit admission, neonatal hypoglycaemia and respiratory distress). The odds ratio for the development of these adverse outcomes remained significant after adjustment for maternal age, body mass index and nonEuropean ethnicity. Those women who were classified as having normal glucose tolerance by WHO criteria but as having GDM by IADPSG criteria also had significant adverse pregnancy outcomes.

Conclusions/interpretation GDM prevalence is higher when using newer IADPSG, compared with WHO, criteria, and these women and their offspring experience significant adverse pregnancy outcomes. Higher rates of GDM pose a challenge to healthcare systems, but improved screening provides an opportunity to attempt to reduce the associated morbidity for mother and child.

Keywords Atlantic DIP. Gestational diabetes mellitus . Maternal and neonatal outcomes · Pregnancy

\begin{tabular}{|c|c|}
\hline \multicolumn{2}{|l|}{ Abbreviations } \\
\hline Atlantic DIP & Atlantic Diabetes In Pregnancy \\
\hline GDM & Gestational diabetes mellitus \\
\hline HAPO & $\begin{array}{l}\text { Hyperglycaemia and Adverse Pregnancy } \\
\text { Outcomes }\end{array}$ \\
\hline IADPSG & $\begin{array}{l}\text { International Association of Diabetes and } \\
\text { Pregnancy Study Groups }\end{array}$ \\
\hline
\end{tabular}

\section{Introduction}

It has long been recognised that overt diabetes diagnosed during pregnancy is associated with significant levels of 
perinatal morbidity, such as macrosomia and neonatal hypoglycaemia [1], but the association between lesser degrees of glucose intolerance and morbidity had not been definitively proven until the publication of the Hyperglycaemia and Adverse Pregnancy Outcomes (HAPO) study [2]. The authors found significant continuous associations between maternal glucose and adverse pregnancy outcomes for mother and offspring at glucose levels below those generally accepted as indicative of overt diabetes [2]. Based on these results and other studies, the International Association of Diabetes and Pregnancy Study Groups (IADPSG) recently issued a consensus statement on new criteria for the diagnosis of gestational diabetes mellitus (GDM) [3]. While the HAPO study has added greatly to our understanding of the significance of even relatively subtle deteriorations in glycaemic control, a significant number of participants were of non-European ethnicity, rendering interpretation of the results in a predominantly European setting difficult.

Accurate up-to-date data on the incidence of hyperglycaemia in pregnancy in Ireland are lacking. It has been over a decade since Irish prevalence figures for overt diabetes of $2.7 \%$ were published [4]. In the intervening time, the prevalence of obesity [5] and immigration of individuals of non-European descent [6] — both risk factors for diabetes mellitus - have increased significantly. The current practice for screening for GDM varies across different centres in Ireland. In the region of our study, the standard practice is to perform a fasting or random glucose test at booking in those with risk factors such as previous GDM, ethnic minority, older age, glycosuria, family history of diabetes mellitus, history of macrosomia or early fetal loss. An OGTT is performed at 24-28 weeks if the initial evaluation is normal. The criteria for diagnosing GDM have, until recently, been based on WHO recommendations [7]. During the period of the study, universal screening was employed.

The aims of our study were to determine the prevalence of GDM using the new criteria in a predominantly European population and to quantify the associated adverse maternal and neonatal outcomes, in particular in those women diagnosed using the newer criteria only.

\section{Methods}

Patient selection Atlantic Diabetes In Pregnancy (DIP) is a multicentre study involving five antenatal centres along the Atlantic coast of Ireland. Established in 2006 following approval from the local ethics board, one of its aims is to determine the prevalence of hyperglycaemia in pregnancy and the associated short- and long-term maternal and fetal outcomes $[8,9]$. Pregnant women whose last menstrual period occurred between September 2006 and March 2009 were eligible to participate in Atlantic DIP. All study participants gave informed consent and full ethical approval was obtained from the local ethics committee.

Diagnostic criteria Atlantic DIP offered universal screening for GDM by means of a $75 \mathrm{~g}$ OGTT at 24-28 weeks. During the course of the study, GDM was diagnosed according to WHO criteria, which classify overt diabetes as fasting and $2 \mathrm{~h}$ glucose $\geq 7.0$ and $\geq 11.1 \mathrm{mmol} / 1$ respectively, and impaired fasting glucose and impaired glucose tolerance as fasting and $2 \mathrm{~h}$ glucose of $6.1-6.9$ and $7.8-11.0 \mathrm{mmol} / \mathrm{l}$ respectively [7]. If GDM was diagnosed, the woman was given advice on dietary and lifestyle modification and instructed in the selfmonitoring of blood glucose. If fasting and $1 \mathrm{~h}$ postprandial glucose targets of $<5.5$ and $<7.8 \mathrm{mmol} / 1$ respectively were not achieved on lifestyle measures alone, insulin therapy was instigated. In order to examine the effect of the application of the new IADPSG criteria on prevalence and outcomes, we applied the cut-off values for a diagnosis of GDM of fasting, 1 or $2 \mathrm{~h}$ glucose $\geq 5.1, \geq 10$ or $\geq 8.5 \mathrm{mmol} / \mathrm{l}$, respectively [3]. In addition we assessed outcomes in women who were diagnosed with GDM on the basis of IADPSG criteria alone (that is, had normal glucose tolerance according to WHO criteria and were therefore not treated as GDM).

Table 1 shows a comparison of the IADPSG, WHO and ADA diagnostic criteria.

Outcomes Various maternal and neonatal outcomes were prospectively recorded. Maternal outcomes recorded were: hypertensive disorders of pregnancy (gestational hypertension and pre-eclampsia); polyhydramnios; mode of delivery (normal vaginal delivery or Caesarean section); and anteand postpartum haemorrhage.

Neonatal outcomes recorded were: live birth rate; congenital malformation rate; premature delivery (defined as delivery before 37 weeks gestation); birthweight; macrosomia (defined as birthweight $>4 \mathrm{~kg}$ ); large for gestational age; small for gestational age; admission to neonatal intensive care unit; duration of stay in neonatal intensive care unit; Apgar score at $5 \mathrm{~min}$; shoulder dystocia; neonatal

Table 1 IADPSG, WHO and ADA criteria for diagnosis of GDM

\begin{tabular}{lccc}
\hline Test & $\begin{array}{l}\text { IADPSG GDM } \\
\text { (any 1 of })\end{array}$ & $\begin{array}{l}\text { ADA GDM } \\
\text { (at least 2 of) }\end{array}$ & $\begin{array}{l}\text { WHO IFG/IGT } \\
\text { (any 1 of })\end{array}$ \\
\hline $\begin{array}{c}\text { Fasting glucose } \\
(\mathrm{mmol} / \mathrm{l})\end{array}$ & $\geq 5.1$ & $\geq 5.3$ & $\geq 6.1$ \\
$\begin{array}{c}\begin{array}{c}\mathrm{h} \text { glucose } \\
(\mathrm{mmol} / \mathrm{l})\end{array} \\
2 \mathrm{~h} \mathrm{glucose} \\
(\mathrm{mmol} / \mathrm{l})\end{array}$ & $\geq 10$ & $\geq 10$ & $\geq 7.8$ \\
\hline
\end{tabular}

${ }^{\mathrm{a}}$ The ADA have recently endorsed the IADPSG criteria [19] 
hypoglycaemia; neonatal jaundice; and neonatal respiratory distress.

Statistical analysis Statistical analysis was carried out using SPSS version 18. Categorical variables were reported as frequencies (\%) and continuous variables were reported using mean (standard deviation) or median (range). Pearson's $\chi^{2}$ test was used to compare the difference in proportions while the independent sample $t$ test was used to compare the difference in the means. A $p$ value of $<0.05$ was taken to signify statistical significance. Logistic regression was used to calculate odds ratios and $95 \%$ confidence intervals for the risk of development of outcomes associated with GDM. A 95\% CI that did not cross 1 was taken as statistically significant. All results were adjusted for potential confounders of maternal age, BMI and ethnicity.

\section{Results}

Prevalence and characteristics of women with GDM A total of 12,487 women were identified and invited to enrol in the study. Of these, 3,237 (25.9\%) refused to participate, and 3,742 (30.0\%) consented but did not attend for their first study visit. This left 5,500 (44.0\%) who completed the study and whose data are presented

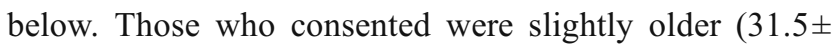
5.5 vs $30.5 \pm 5.8$ years, $p<0.0001)$ and more overweight (BMI of $26.9 \pm 5.1$ vs $25.9 \pm 4.5 \mathrm{~kg} / \mathrm{m}^{2}, p<0.0001$ ) than those who did not, but there was no difference in ethnicity.

The mean $( \pm \mathrm{SD})$ age was $31.5 \pm 5.5$ years, mean $( \pm \mathrm{SD})$ BMI was $26.9 \pm 5.1 \mathrm{~kg} / \mathrm{m}^{2}$, and median (range) parity and gravida were 1 (0-9) and 2 (1-14), respectively. There were $391(7.1 \%)$ participants of non-European descent. There was a history of diabetes in a first or second degree relative in $1,758(32.0 \%)$ and there was a history of previous early fetal loss in $1,256(23 \%)$.

Glucose results Median (range) fasting, 1 and $2 \mathrm{~h}$ glucose values on OGTT were 4.3 (3-12.2), 6.8 (2.4-17.5) and 5.3 (1.8-18.7) mmol/1, respectively. Using the new (IADPSG) criteria, 680 participants (12.4\%) had GDM, compared with $520(9.4 \%)$ according to WHO criteria. IADPSG criteria for GDM were satisfied in $394(7.2 \%)$ women on fasting glucose, $342(6.3 \%)$ at $1 \mathrm{~h}$, and $249(4.6 \%)$ at $2 \mathrm{~h}$. There were 204 women $(3.7 \%)$ with a fasting glucose diagnostic for IADPSG GDM, but with normal 1 and $2 \mathrm{~h}$ glucose levels. Similarly, there were 142 women $(2.6 \%)$ in whom IADPSG GDM was diagnosed based on $1 \mathrm{~h}$ glucose alone and $77(1.4 \%)$ based on $2 \mathrm{~h}$ glucose alone. A diagnosis of overt diabetes based on a fasting glucose level of $\geq 7.0 \mathrm{mmol} / \mathrm{l}$ was reached in 25 women ( $0.5 \%$ ), but markedly elevated 1 and $2 \mathrm{~h}$ glucose levels $\geq 11.1 \mathrm{mmol} / 1$ were present in $176(3.3 \%)$ and 46 $(0.8 \%)$ respectively. There were 13 women $(0.2 \%)$ in whom overt diabetes was based on fasting glucose alone, $129(2.3 \%)$ on $1 \mathrm{~h}$ glucose alone and $11(0.2 \%)$ on $2 \mathrm{~h}$ glucose alone.

Of the 520 women with WHO-defined GDM, 93 (17.9\%) required insulin in addition to dietary modification to achieve adequate glycaemic control. Of these women, 33\% were prescribed with a long-acting insulin (isophane), and 66\% were prescribed with short-acting insulin analogues (Actrapid [Novo Nordisk] or aspart [B28Asp human insulin]). Mean (range) first measured $\mathrm{HbA}_{1 \mathrm{c}}$ was $5.6(4.6-6.9) \%$; mean (range) week of measurement was 29.2 (17-37) weeks of gestation.

Those with IADPSG-defined GDM were older (mean age $32.9 \pm 5.3$ vs $31.3 \pm 5.4$ years, $p<0.0001$ ), more overweight (mean BMI $30.1 \pm 6.4$ vs $26.6 \pm 4.8 \mathrm{~kg} / \mathrm{m}^{2}, p<0.0001$ ), of a higher gravity $(2.67 \pm 1.79$ vs $2.26 \pm 1.47, p<0.0001)$ and parity $(1.22 \pm 1.39$ vs $0.94 \pm 1.12, p<0.0001)$, more likely to be of non-European descent $(16.7 \%$ vs $16.7 \%, p<0.0001)$, have a positive family history $(47.4 \%$ vs $29.9 \%, p<0.0001)$ and previous miscarriage ( $31.1 \%$ vs $24.2 \%, p=0.01)$.

Adverse maternal outcomes Table 2 shows the prevalence of the maternal adverse outcomes recorded and Table 3 the associated ORs (95\% CI) for developing these complications associated with a diagnosis of IADPSG-defined GDM. There were significantly more cases (and associated increased odds ratios) for gestational hypertension, polyhydramnios and Caesarean section rates in women with GDM. While there were more cases of pre-eclampsia and fewer normal vaginal deliveries in the GDM group, the odds ratios for these variables did not reach statistical significance. There was no difference in rates of ante- or post-partum haemorrhage (data not shown). The majority of Caesarean sections in the GDM group were elective $(n=132$; $53.7 \%$ ), but emergency in the normal glucose tolerance group ( $n=621 ; 53.3 \%), p=0.04$. The increased odds of Caesarean section associated with GDM remained significant after adjustment for hypertensive disorders of pregnancy.

Table 2 Prevalence of maternal outcomes associated with a diagnosis of IADPSG-defined GDM

\begin{tabular}{lccc}
\hline Variable & NGT & GDM & $p$ value \\
\hline Gestational hypertension & $332(7.5)$ & $86(13.8)$ & $<0.0001$ \\
Pre-eclampsia & $176(4.0)$ & $39(6.3)$ & 0.007 \\
Polyhydramnios & $37(0.8)$ & $21(3.4)$ & $<0.0001$ \\
Normal vaginal delivery & $2,708(57.9)$ & $340(51.4)$ & 0.002 \\
Caesarean section & $1,165(24.9)$ & $246(37.2)$ & $<0.0001$ \\
\hline
\end{tabular}

Values are $n(\%)$

NGT, normal glucose tolerance 
Table 3 ORs and 95\% CIs for maternal complications associated with IADPSG-defined GDM

\begin{tabular}{lcc}
\hline Variable & OR & $95 \%$ CI \\
\hline Gestational hypertension & 1.5 & $1.0-2.0$ \\
Pre-eclampsia & 1.1 & $0.7-1.8$ \\
Polyhydramnios & 2.5 & $1.2-5.2$ \\
Normal vaginal delivery & 0.8 & $0.7-1.1$ \\
Caesarean section & 1.3 & $1.0-1.6$ \\
\hline
\end{tabular}

Adverse neonatal outcomes Table 4 shows the prevalence of the neonatal adverse outcomes recorded and Table 5 the ORs $(95 \% \mathrm{CI})$ for those outcomes that were found to be statistically significant in Table 4 . There was no difference in the live birth or congenital malformation rates between the two groups. An earlier mean week of delivery translated into higher rates of prematurity with a $70 \%$ increased risk in women with GDM. Although there was no difference in mean birthweight and only borderline increased rate of macrosomia, there was a $30 \%$ and $60 \%$ increased odds ratio for large and small for gestational age, respectively, in women with GDM. After adding smoking history, hypertensive disorders of pregnancy and insulin use to the statistical model, GDM was no longer a predictor of small for gestational age babies, but smoking was independently associated with the condition; OR 4.0 (1.0-15.9), $p=0.05$.

Babies born to mothers with GDM were almost four times more likely to be admitted to a neonatal intensive care unit, though their length of stay was significantly shorter. Neonatal hypoglycaemia and respiratory distress were
Table 5 ORs and 95\% CIs for neonatal complications associated with IADPSG-defined GDM

\begin{tabular}{lcc}
\hline Variable & OR & $95 \%$ CI \\
\hline Premature delivery & 1.7 & $1.1-2.6$ \\
Macrosomia & 1.2 & $0.7-2.1$ \\
Large for gestational age & 1.3 & $1.0-1.7$ \\
Small for gestational age & 1.6 & $1.0-2.5^{\mathrm{a}}$ \\
Neonatal intensive care unit admission & 3.9 & $3.0-5.1$ \\
Neonatal hypoglycaemia & 3.4 & $1.3-9.0$ \\
Neonatal respiratory distress & 2.0 & $1.1-3.7$ \\
\hline
\end{tabular}

${ }^{\mathrm{a}}$ After correction for smoking history, hypertensive disorders of pregnancy and insulin use, the value no longer remained statistically significant

significantly more likely in the GDM group, but there were no differences in Apgar scores, or rates of shoulder dystocia or neonatal jaundice.

There were 258 women who were diagnosed with GDM using IADPSG criteria but normal glucose tolerance using WHO criteria. Thus these women were untreated and were therefore similar to participants in studies by Crowther et al. [10] and Landon et al. [11]. Compared with IADPSG criteria-defined normal glucose tolerance, these women had higher incidence of gestational hypertension ( $15 \%$ vs $7.5 \%$, $p<0.0001)$, pre-eclampsia $(7.1 \%$ vs $4.0 \%, p=0.03)$, and Caesarean section rates $(35.2 \%$ vs $24.9 \%, p<0.0001)$. In terms of neonatal outcomes, there were higher rates of macrosomia $(28.8 \%$ vs $17.0 \%, p=0.02)$, large for gestational age $(26.8 \%$ vs $16.2 \%, p<0.0001)$ and neonatal intensive care unit admission $(16.5 \%$ vs $9.1 \%, p<0.0001)$ in this group. There were no other differences in maternal
Table 4 Prevalence of neonatal outcomes associated with a diagnosis of IADPSG-defined GDM

Values are $n(\%)$ or mean \pm SD unless stated otherwise

\begin{tabular}{llll}
\hline Variable & NGT & GDM & $p$ value \\
\hline Live births & $4,662(99.5)$ & $659(99.1)$ & NS \\
Congenital malformations & $70(1.5)$ & $15(2.3)$ & NS \\
Week of delivery & $39.4 \pm 1.88$ & $38.8 \pm 2.4$ & $<0.0001$ \\
Premature delivery & $223(4.8)$ & $47(7.1)$ & 0.002 \\
Birthweight & $3.6 \pm 0.5$ & $3.5 \pm 0.6$ & $\mathrm{NS}$ \\
Macrosomia & $156(17)$ & $33(23.9)$ & 0.05 \\
Large for gestational age & $751(16.2)$ & $149(22.6)$ & $<0.0001$ \\
Small for gestational age & $203(4.4)$ & $38(5.8)$ & $\mathrm{NS}$ \\
Neonatal intensive care unit admission & $419(9.1)$ & $170(26.0)$ & $<0.0001$ \\
Days in neonatal intensive care unit, & $5.0(1-93)$ & $3.0(1-47)$ & $<0.0001$ \\
median (range) & $13(0.3)$ & $2(0.3)$ & $\mathrm{NS}$ \\
Apgar $<7$ at 5 min & $57(1.2)$ & $8(1.2)$ & $\mathrm{NS}$ \\
Shoulder dystocia & $28(0.6)$ & $16(2.4)$ & $<0.0001$ \\
Neonatal hypoglycaemia & $316(6.7)$ & $39(5.8)$ & $\mathrm{NS}$ \\
Neonatal jaundice & $86(1.8)$ & $24(3.6)$ & 0.002 \\
Neonatal respiratory distress & &
\end{tabular}


or neonatal outcomes in this group compared with women with IADPSG-defined normal glucose tolerance.

\section{Discussion}

Using the new IADPSG criteria we identified a prevalence rate of GDM of $12.4 \%$ in a mainly European population. This was higher than the rate of $9.4 \%$ that was observed when WHO criteria (with higher fasting and $1 \mathrm{~h}$, but lower $2 \mathrm{~h}$ glucose cut-offs) were applied. Worldwide, reported prevalence rates of GDM depend on which diagnostic criteria are used and have ranged from $1 \%$ to $14 \%$ [12]. It has been estimated that, if the new criteria were applied to the HAPO cohort, the prevalence of GDM (including overt diabetes) would be $17.8 \%$ [3]. Reasons for the lower rate in our study compared with the study by Metzger et al. [2] may include a higher European-origin population $(92.9 \%$ compared with $48.3 \%)$ and lower mean BMI $(26.9 \pm 5.1$ compared with $27.7 \pm 5.1 \mathrm{~kg} / \mathrm{m}^{2}$ ). A recent study comparing IADPSG to ADA criteria found a threefold increase with the newer criteria, although this was in a high-risk ethnic group [13]. The application of these new diagnostic criteria for GDM will result in a greater number of women receiving the diagnosis than with the previous criteria, which will in turn have a significant impact on manpower and financial resources.

A limitation of our study was that the uptake was $44 \%$. Those who refused or did not attend after initially consenting were statistically slightly younger and of a lower BMI, but there was no difference in ethnicity. It is possible, therefore, that the actual prevalence within the population as a whole may be slightly lower than the $12.4 \%$ identified in our study. However, it is important to note that complete data were not available for all the women who did not participate and the absolute differences seen were relatively small. We feel that, based on these findings, our sample is representative of the population at large in the region of our study.

A diagnosis of GDM was associated with a significant number of adverse maternal and neonatal outcomes. In the mother, there was a $30 \%, 50 \%$ and $250 \%$ increased risk of requiring a Caesarean section, or developing gestational hypertension or polyhydramnios, respectively. In the offspring, risk of prematurity was increased by $70 \%$, large and small for gestational age by $30 \%$ and $60 \%$ respectively, and newborns were $4,3.5$ and 2 times more likely to be admitted to neonatal intensive care units, or develop neonatal hypoglycaemia or respiratory distress, respectively. Comparison of these outcomes with other studies assessing outcomes in women at levels of dysglycaemia similar to the new criteria is difficult. Unlike our study, Metzger et al. [2], Crowther et al. [10] and Landon et al. [11] excluded women with greater degrees of hyperglycaemia. Considering this, the prevalence of the various maternal adverse events is similar to the 'routine care' groups in the Crowther et al. [10] and Landon et al. [11] studies. As expected, there were significantly fewer events in the HAPO study as these were women with lesser degrees of glucose intolerance than in our study [2]. An interesting finding is that of a higher Caesarean section rate in women with GDM, mainly as a result of more elective sections being performed. It is well accepted that GDM increases risk of Caesarean section [1], but there is also evidence that this risk may be further increased by the coexistence of hypertensive disorders of pregnancy [14]. However, even after adjusting for those with hypertension, GDM was still predictive of Caesarean section.

In terms of neonatal outcomes, while increased risk of prematurity [15] and being large for gestational age [16] are well-recognised in association with GDM, the $60 \%$ increased risk of being small for gestational age was an interesting finding. There are multiple causes for being small for gestational age, including diabetes [17]. In order to clarify whether there was a confounding cause for this finding we added smoking history, the presence of a hypertensive disorder in pregnancy, and insulin use to our logistic regression model. We included insulin use as it has been suggested by some that excessive control of hyperglycaemia with insulin could result in reduced intrauterine fetal growth [18]. After adjustment for all these factors, we found that GDM was no longer predictive of small for gestational age, but smoking was associated with a fourfold increased risk, $p=0.05$. The higher rate of neonatal intensive care unit admission in neonates of GDM women was associated with shorter stays. This raises the possibility that these neonates were admitted there 'for observation' rather than for specific medical indications - a practice that could be employed by some centres covering large rural areas. The earlier week of delivery in the women with GDM may, in part, reflect the tendency to induce labour rather than letting term be reached, to avoid further intra-uterine growth, but the finding is in keeping with that of the HAPO study, which found an association between increasing maternal glucose levels and prematurity [2].

We also wished to evaluate whether women who were classified as having GDM by IADPSG criteria but normal glucose tolerance by WHO criteria had any worse outcomes than women with IADPSG-defined normal glucose tolerance. We found higher rates of hypertensive complications of pregnancy, Caesarean section, macrosomia, large for gestational age and neonatal intensive care unit admission. In keeping with the findings of Metzger et al. [2], upon which the new criteria are partly based, we 
found adverse outcomes in these women of relatively 'mild' hyperglycaemia.

There are a number of limitations to our study. It was conducted over five centres, and obstetric practice may differ slightly. However, this is indicative of routine clinical practice and makes the results more applicable to real-life clinical care. During the course of the study, the WHO criteria were used to diagnose GDM and decisions on treatment were based on this classification. Our application of the new criteria (which were published after the study was complete) makes it impossible to comment on the effect of hyperglycaemia treatment on outcomes in the study. Examining the effect of hyperglycaemia management was not an aim of the study and the application of the new criteria allows conclusions to be drawn on what outcomes are associated with these pregnancies in a less ethnically heterogeneous population than that of the HAPO Study [2].

There are a number of important implications for future practice raised by this study. New criteria will result in a greater number of women being diagnosed with GDM. The higher Caesarean section and neonatal intensive care rates seen in GDM, independent of typical causative factors, raises the possibility that these women and neonates may be being treated differently as a result of the diagnostic label of GDM. While this may be appropriate in the majority of cases, the increasing numbers of women who will be diagnosed with the new criteria will put health systems under significant pressure and careful thought should be given, in each individual case, to whether Caesarean section and neonatal intensive care is really indicated.

Women who fulfilled the IADPSG criteria for GDM, but were of normal glucose tolerance using WHO criteria, experienced significantly higher adverse pregnancy outcomes, suggesting that the application of these new criteria is applicable to a mainly European population.

The identification of greater numbers of women with GDM using the new criteria will pose a significant challenge to healthcare systems already under pressure from financial and personnel perspectives, but it also affords an opportunity to diagnose these women and hopefully reduce the associated morbidity for mother and child.

Acknowledgements Funding for the study was provided by the Health Research Board of Ireland.

Duality of interest The authors declare that there is no duality of interest associated with this manuscript.

\section{References}

1. Hod M, Merlob P, Friedman S, Schoenfeld A, Ovadia J (1991) Gestational diabetes mellitus. A survey of perinatal complications in the 1980s. Diabetes 40(Suppl 2):S74-S78

2. Metzger BE, Lowe LP, Dyer AR et al (2008) Hyperglycemia and adverse pregnancy outcomes. N Engl J Med 358:1991-2002

3. International Association of Diabetes and Pregnancy Study Groups Consensus Panel (2010) International Association of Diabetes and Pregnancy Study Groups recommendations on the diagnosis and classification of hyperglycemia in pregnancy. Diabetes Care 33:676-682

4. Griffin ME, Coffey M, Johnson H et al (2000) Universal vs risk factor-based screening for gestational diabetes mellitus: detection rates, gestation at diagnosis and outcome. Diabet Med 17:26-32

5. Harrington J, Perry I, Lutomski J et al (2008) SLÁN 2007: Survey of lifestyle, attitudes and nutrition in Ireland. Dietary Habits of the Irish Population, Department of Health and Children. The Stationery Office, Dublin

6. Central Statistics Office (2009) Population and migration estimates April 2009. Central Statistics Office, Dublin

7. World Health Organization/International Diabetes Federation (2006) Definition and diagnosis of diabetes mellitus and intermediate hyperglycemia: report of a WHO/IDF consultation. WHO, Geneva

8. Dunne FP, Avalos G, Durkan M et al (2009) ATLANTIC DIP: pregnancy outcome for women with pregestational diabetes along the Irish Atlantic seaboard. Diabetes Care 32:1205-1206

9. Owens LA, O'Sullivan EP, Kirwan B, Avalos G, Gaffney G, Dunne F (2010) ATLANTIC DIP: the impact of obesity on pregnancy outcome in glucose-tolerant women. Diabetes Care 33:577-579

10. Crowther CA, Hiller JE, Moss JR, McPhee AJ, Jeffries WS, Robinson JS (2005) Effect of treatment of gestational diabetes mellitus on pregnancy outcomes. N Engl J Med 352:2477-2486

11. Landon MB, Spong CY, Thom E et al (2009) A multicenter, randomized trial of treatment for mild gestational diabetes. N Engl J Med 361:1339-1348

12. Jovanovic L, Pettitt DJ (2001) Gestational diabetes mellitus. JAMA 286:2516-2518

13. Agarwal MM, Dhatt GS, Shah SM (2010) Gestational diabetes mellitus: simplifying the international association of diabetes and pregnancy diagnostic algorithm using fasting plasma glucose. Diabetes Care 33:2018-2020

14. Stella CL, O'Brien JM, Forrester KJ et al (2008) The coexistence of gestational hypertension and diabetes: influence on pregnancy outcome. Am J Perinatol 25:325-329

15. Ostlund I, Hanson U, Bjorklund A et al (2003) Maternal and fetal outcomes if gestational impaired glucose tolerance is not treated. Diabetes Care 26:2107-2111

16. Langer O (2000) Fetal macrosomia: etiologic factors. Clin Obstet Gynecol 43:283-297

17. Lee PA, Chernausek SD, Hokken-Koelega AC, Czernichow P (2003) International Small for Gestational Age Advisory Board consensus development conference statement: management of short children born small for gestational age, April 24-October 1, 2001. Pediatrics 111:1253-1261

18. Rosenn BM, Miodovnik M (2000) Glycemic control in the diabetic pregnancy: is tighter always better? J Matern Fetal Med 9:29-34

19. American Diabetes Association (2010) Diagnosis and classification of diabetes mellitus. Diabetes Care 34(Suppl 1):S15 